\title{
CARACTERIZAÇÃO DAS VÍTIMAS E DOS ACIDENTES DE TRABALHO FATAIS
}

\author{
Fernanda Moura D’Almeida MIRANDAª Louise Aracema SCUSSIATO ${ }^{b}$, Ana Lúcia Cardoso KIRCHHOF ,
} Elaine Drehmer de Almeida CRUZ ${ }^{\text {, }}$ Leila Maria Mansano SARQUIS ${ }^{e}$

\section{RESUMO}

Trata-se de uma pesquisa documental, quantitativa, descritiva, retrospectiva sobre os acidentes de trabalho fatais ocorridos no período de 2006 a 2010 e cujos trabalhadores foram atendidos no Hospital do Trabalhador, localizado em Curitiba/Paraná. Foram selecionadas 25 fichas de notificação pelo desfecho óbito. Objetivou-se caracterizar as vítimas e os acidentes de trabalho fatais. Os acidentes de trabalho fatais atingiram trabalhadores com idade média de 35 anos (desvio padrão=13,0694), do gênero masculino $n=23(92 \%)$. Os acidentes de trabalho típicos representaram $n=13$ (52\%) dos casos. Uma das medidas preventivas propostas para reduzir esse número de acidentes de trabalho fatais é a realização de um trabalho educativo e preventivo nos ambientes de trabalho, pelos profissionais enfermeiros. Além disso, é necessário repensar a violência no trânsito também como um fator relevante de causa de morte de trabalhadores.

Descritores: Acidentes de trabalho. Mortalidade ocupacional. Notificação de acidentes de trabalho. Saúde do trabalhador.

\section{RESÚMEN}

Esta es una investigación cuantitativa descriptiva documental retrospectiva sobre los accidentes mortales se produjeron durante el período 2006 a 2010 y los trabajadores fueron tratados en el Hospital del Trabajador, ubicado en Curitiba / Paraná. Se seleccionaron 25 fichas de notificación por los resultados obtenidos. Se objetivo caracterizar a las víctimas y los accidentes de trabajo faltaless. Los accidentes laborales fatalçes alcanzaron a trabajadores coe edad promedio de 35 años (desvio patrón= 13,0694), del género masculino $n=23$ (92\%). Los accidentes laborales típicos representaron n=13 (52\%) de los casos. Una de las medidas preventivas propuestas para reducir ese número de accidentes de trabajo fatales es la realización de um trabajo educativo y preventivo en los ambientes de trabajo, por los profesionales enfermeros. Además, es necesario repensar la violencia en el tránsito también un factor relevante de causa de muerte de trabajadores.

Descriptores: Accidentes de trabajo. Mortalidad laboral. Notificación de accidentes del trabajo. Salud laboral. Título: Características de las víctimas y accidentes fatales en el trabajo.

\section{ABSTRACT}

This is a quantitative, descriptive, retrospective documental study on fatal accidents occurred during the period from 2006 to 2010, in which workers were treated at Hospital do Trabalhador, located in Curitiba/Paraná. We selected 25 notifications for the outcome death. This study aimed to characterize victims and fatal occupational accidents. Fatal occupational accidents hit workers with a mean age of 35 years (SD =13.0694), of the male sex, $n=23$ (92\%). Typical occupational accidents accounted for $52 \%(n=13)$ of cases. One of the preventive measures proposed to reduce the number of fatal occupational accidents is the performance of educational and preventive work at the workplace by nurses. Moreover, it is necessary to rethink transit violence as a relevant factor for the cause of death of workers as well.

Descriptors: Accidents, Occupational. Occupational mortality. Occupational accidents registry. Occupational health. Title: Characteristics of victims and fatal accidents at the workplace.

a Enfermeira. Mestre em Enfermagem no Programa de Pós-Graduaçao em Enfermagem da Universidade Federal do Paraná (PPGENF/UFPR). Coordenadora Operacional da Unidade Saúde do Trabalhador do Hospital do Trabalhador (UST/HT). Coordenadora da Pós-Graduação em Enfermagem do Trabalho da Universidade Positivo em Curitiba/Paraná/Brasil. Membro do Grupo de Pesquisa em Saúde do Adulto (GEMSA/UFPR).

b Enfermeira. Mestranda em Enfermagem no Programa de Pós-Graduação em Enfermagem da Universidade Federal do Paraná (PPGENF/ UFPR) em Curitiba/Paraná/Brasil. Bolsista Coordenação de Aperfeiçoamento de Pessoal de Aperfeiçoamento de Pessoal de Nível Superior (CAPES). Membro do Grupo de Pesquisa GEMSA.

c Enfermeira. Doutora em Enfermagem. Pesquisadora visitante do Programa de Pós Graduação em Enfermagem da UFPR 2008-2010 e professora aposentada do Departamento de Enfermagem e do Programa da Pós - Graduação da Universidade Federal de Santa Catarina. Florianópolis/ Santa Catarina/Brasil.

d Enfermeira, Doutora em Enfermagem. Professora do Curso de Graduação e do PPGENF UFPR. Membro do GEMSA/UFPR em Curitiba/ Paraná/Brasil.

e Enfermeira, Doutora em Enfermagem. Professora do Curso de Graduação e do PPGENF UFPR, em Curitiba/Paraná/Brasil. Vice-líder do Grupo de Pesquisa GEMSA, Membro do Grupo de Pesquisa de Qualidade de Vida do Departamento ENO da EEUSP. 


\section{INTRODUÇÃO}

As ações para a prevenção e enfrentamento dos acidentes de trabalho (AT) no Brasil são preconizadas pelo Sistema de Único de Saúde (SUS), em conjunto com órgãos do serviço público e da sociedade civil. Os AT típicos podem ocorrer na execução das atividades laborais e os AT de trajeto durante o percurso que o trabalhador realiza da sua residência ao trabalho, ou vice-versa ${ }^{(1)}$.

Os AT típicos assim como os AT de trajeto podem ocasionar, inclusive, o óbito. Porém, os AT são evitáveis por meio de políticas públicas e institucionais que favoreçam a sua prevenção através da neutralização ou eliminação de fatores capazes de desencadeá-los. Esses fatores ou causas são classificados como preveníveis quando passíveis de serem afastados os riscos como: condições de trabalho inadequadas, falta de conhecimento e qualificação profissional ou máquinas inseguras, entre outras. Já as causas imprevisíveis nos AT são aqueles que, apesar de serem tomadas medidas para evitá-las, não dependem do trabalhador e nem do empregador ${ }^{(2)}$.

No Brasil, a primeira Lei sobre AT foi aprovada em 1919, porém restringia o acidente como sendo causado única e exclusivamente pela atividade laboral; a partir de 1944 a inclusão do conceito concausalidade permitiu maior amplitude para o conceito de AT. Porém, somente, em 1967 com a Lei $\mathrm{n}^{\mathrm{o}} 5.316$ foi incorporado o termo de AT ocorrido no trajeto para o trabalho e também o reconhecimento das doenças do trabalho, bem como a obrigatoriedade das empresas em emitir a Comunicação de Acidente de Trabalho (CAT) para os segurados pelo Instituto Nacional de Previdência Social (INSS) ${ }^{(3)}$. Em 2004 o Ministério da Saúde (MS) tornou compulsória a notificação de AT graves/fatais, inclusive dos trabalhadores do mercado informal, por meio do Sistema Nacional de Notificação de Agravos (SINAN), determinação essa reiterada pela Portaria do Gabinete Ministerial/Ministério da Saúde $n^{\circ}$ $2472 \mathrm{em} 2010^{(4-5)}$.

O SINAN auxilia significativamente a vigilância às doenças relacionadas ao trabalho, entre elas, os AT fatais, e tem como objetivo coletar dados que serão analisados e utilizados para desenvolver projetos e ações em saúde do trabalhador no contexto das políticas públicas de saúde. Este sistema apresenta abrangência no registro de casos de agravos relacionados ao trabalho, pois insere tanto os trabalhadores segurados como os servidores públicos, trabalhadores autônomos e desempregados, entre outros ${ }^{(6)}$.

Para salientar a importância desta temática destaca-se que no Brasil, em 2009, foram registrados cerca de 750 mil AT entre os trabalhadores segurados pelo Instituto Nacional de Previdência Social (INSS), sendo que ocorreram 2851 AT fatais. Em outras palavras, ocorreu naquele ano uma morte a cada três horas; em média 31 trabalhadores ao dia não retornaram às atividades de trabalho por invalidez ou morte. Estes dados mostram apenas a realidade dos trabalhadores do mercado formal, ou seja, segurados pelo INSS; calcula-se que metade da população economicamente ativa brasileira esteja na informalidade ${ }^{(5,7)}$. Desse modo, destaca-se a importância do SINAN para o conhecimento da real dimensão do problema dos AT em nosso país.

Apesar da legislação e ações de prevenção os AT fatais ocorrem e causam prejuízos pessoais, sociais, econômicos às famílias e à sociedade. São elevados custos para a economia nacional, tanto de forma direta, pelos custos assistenciais e previdenciários, quanto de forma indireta, representados pela perda da força de trabalho e do investimento na formação do cidadão ${ }^{(8)}$. Considerando a magnitude desta temática objetivou-se neste estudo caracterizar as vítimas e os acidentes de trabalho fatais.

\section{METODOLOGIA}

Trata-se de pesquisa documental, quantitativa, descritiva e retrospectiva realizada no primeiro trimestre de 2011, por meio da coleta de dados nas fichas eletrônicas de notificação de AT no SINAN. O período do estudo foi de agosto de 2006 a dezembro de 2010. Em agosto de 2006 foi implantado o SINAN na unidade sentinela do Hospital do Trabalhador (HT), denominada Unidade Saúde do Trabalhador (UST), localizado no município de Curitiba/ Paraná permitindo assim a coleta retrospectiva das informações.

Para a coleta de dados foram consultadas as 1.747 fichas de notificação de AT graves cujos trabalhadores foram atendidos no HT e os casos notificados ao SINAN pela UST. Foi critério de inclusão no estudo os casos de AT com desfecho de óbito relacionado ao AT, totalizando 25 casos. Os dados coletados foram idade, sexo, situação no mercado de trabalho, emissão de CAT, tipo de acidente, ocupação, causa do acidente e parte do corpo atingida. Após coletados, foram registrados 
em planilha eletrônica e submetidos a tratamento estatístico descritivo para análise. Os dados são apresentados por meio da freqüência dos dados e em forma de tabelas e gráfico.

Este estudo foi aprovado pelo Comitê de Ética em Pesquisa da Secretaria Estadual de Saúde do Paraná/ Hospital do Trabalhador (CEP-SESA/HT) sob Certificação de Apresentação para Apreciação Ética número 0015.0.429.091-10 e sob o protocolo do CEP-SESA/HT n'231/2010, o qual teve assegurado o caráter de anonimato dos registros utilizados dos casos notificados pelo SINAN. O HT autorizou a divulgação do nome da instituição.

\section{RESULTADOS}

Entre os 25 casos de AT fatais, 13(57\%) acometeram trabalhadores adultos jovens com idade entre 19 e 30 anos. Em relação ao gênero os casos analisados foram 23(92\%) do sexo masculino. A idade dos trabalhadores variou entre 19 a 64 anos, como demonstra a Tabela 1 .

De acordo com a situação no mercado de trabalho a maioria dos trabalhadores - 17 (68\%) encontravam-se em emprego com carteira assinada, 2(8\%) eram servidores públicos estatutários, o que representa $76 \%$ no mercado formal da economia, e $1(4 \%)$ autônomo e $3(12 \%)$ empregados sem registro, representado (16\%) no mercado informal. Em 19(76\%) dos casos foi emitida a CAT devido à maioria dos trabalhadores pertencerem ao mercado formal da economia.

Ao analisar o tipo de AT verificou-se que ocorreram 13 (52\%) de AT fatais típicos enquanto os AT fatais de trajeto totalizaram 11 (44\%), sendo apenas um caso registrado como ignorado.

Na Tabela 2 as ocupações estão classificadas segundo ramo de atividade que consta da Norma

Tabela 1 - Caracterização das vítimas de acidente de trabalho fatal, segundo faixa etária. Curitiba. SINAN-UST/HT, 2006-2010

\begin{tabular}{lll}
\hline FAIXA ETÁRIA* & $\begin{array}{l}\text { Frequência } \\
\text { Absoluta }\end{array}$ & \% \\
\hline 19 a 30 anos & 13 & 52,0 \\
31 a 45 anos & 5 & 20,0 \\
46 a 60 anos & 4 & 16,0 \\
61 a 64 anos & 1 & 4,0 \\
\hline TOTAL & $\mathbf{2 5}$ & $\mathbf{1 0 0 , 0}$ \\
\hline
\end{tabular}

*Desvio padrão $=13,0694$

Tabela 2 - Caracterização das vítimas de AT fatais, segundo ocupação por ramo de atividade. Curitiba. SINAN-UST/HT, 2006-2010

\section{OCUPAÇÃO POR RAMO DE ATIVIDADE}

Comércio, reparação de veículos automotores, objetos pessoais e domésticos (auxiliar de escritório, lavador de veículos, mecânico, operador de empilhadeira e supervisor administrativo)

Transporte, armazenagem e comunicação (ajudante de motorista, motociclista de transporte de documentos e pequenos volumes e motorista de caminhão)

Construção (pedreiro, desenhista projetista de arquitetura)

Atividades imobiliárias, aluguéis e serviços prestados às empresas (corretor de imóveis, trabalhador de serviços de manutenção de edifícios e logradouros e jardineiro)

Indústria (apontador de produção e operador de máquina)

Agricultura, pecuária, silvicultura e exploração florestal (operador de motosserra)

Administração Pública, defesa e seguridade social (guarda municipal)

Ignorado

Frequência
Absoluta

06 
Regulamentadora (NR) n⿳4十 da Portaria n 3214 de o8 de junho de $1978^{(14)}$.

Verifica-se na Tabela 3 que os acidentes envolvendo colisão com veículos a motor foi a causa externas de $10(40 \%)$ AT fatais, seguido de queda de nível com 5 (20\%).

Como podemos observar no Gráfico 1 que 14 (56\%) dos óbitos foi consequência de ferimentos na cabeça provocados por acidentes de trânsito e quedas.

\section{DISCUSSÃO}

Este estudo evidenciou que a maioria dos acidentes atingiu homens jovens e produtivos com elevada participação na força de trabalho, e em especial, em atividades de maior grau de risco. Tal qual este estudo, a construção civil, seguido dos transportes são os ramos de atividade produtiva nos quais ocorrerem o maior número de AT fatais, ainda apontou a ocorrência de elevada mortalidade entre trabalhadores com idade até 30 anos e do sexo masculino e também que o coeficiente de mortalidade é oito vezes maior para os homens em relação às mulheres ${ }^{(9)}$. Em outro estudo realizado no Vale dos Sinos no estado do Rio Grande do Sul, em 2008, evidenciou que $64 \%$ dos acidentes de trabalho atingiram homens, $38 \%$ na faixa etária de 20 a 29 anos e $83,7 \%$ dos acidentes ocorreram no trabalho. Outro dado apontando é que $60 \%$ dos trabalhadores eram registrados ${ }^{(10)}$. Outro estudo realizado em Salvador/ Bahia, em 2008 apontou que a maioria dos casos ocorreram no sexo masculino $(77,8 \%)$, idade acima de 28 anos $(69,7 \%)$, com predominância de trabalhadores do ramo de transportes e comércio. Isso mostra que os homens, adultos jovens são as maiores vítimas dos acidentes fatais no Brasil e que essa realidade está evidenciada por outros estudos e ainda permanece inalterada ${ }^{(11)}$.

Em relação à situação no mercado de trabalho, a maioria dos trabalhadores que sofreram AT fatais encontrava-se no setor formal da economia. Este dado difere de outro estudo ${ }^{12}$ realizado no Rio Grande do Sul, em 2008, a informalidade no trabalho foi prevalente entre os trabalhadores com maior número de óbitos. Os trabalhadores informais estão desamparados tanto na parte jurídica quanto assistencial por não estarem assegurados pela previdência social. Considera-se, portanto, que o fato dos trabalhadores e seus familiares estarem assegurados em caso de acidentes fatais, possibilita a busca por indenizações e outros direitos que possam remediar esse terrível agravo. Os trabalhadores que possuem carteira assinada e contribuição ao INSS têm assegurados seus direitos previdenciários.

Tabela 3 - Caracterização dos AT fatais segundo causa do acidente. Curitiba. SINAN-UST/HT, 2006-2010.

\section{CAUSA DO ACIDENTE}

Queda de nível ou para fora de edifícios

Projétil de revólver

Motociclista traumatizado em colisão com um veículo de transporte pesado ou um ônibus e/ou em acidente de transporte sem colisão

Impacto causado por objeto lançado, projetado ou em queda

Ciclista traumatizado em colisão com um automóvel, "pick up" ou caminhonete, veículo pesado ou ônibus e/ ou veículo motor de duas ou três rodas

Apertado, colhido, comprimido ou esmagado dentro de ou entre objetos

Pedestre traumatizado em outros acidentes de transporte e em acidentes de transporte não especificados ou colisão com uma automóvel [carro], "pick up" ou caminhonete

Ocupante de um automóvel [carro] traumatizado em colisão com um objeto fixo ou parado ou em colisão com um automóvel [carro], "pick up" ou caminhonete

Contato com outras máquinas e com as não especificadas

Circunstância relativa às condições de trabalho

\begin{tabular}{ll}
$\begin{array}{l}\text { Frequencia } \\
\text { Absoluta }\end{array}$ & \% \\
\hline 05 & 20 \\
03 & 12 \\
03 & 12 \\
02 & 08 \\
04 & 16 \\
02 & 08 \\
2 & 8 \\
01 & \\
01 & 04 \\
01 & 04 \\
\hline $\mathbf{2 5}$ & 04 \\
\hline
\end{tabular}




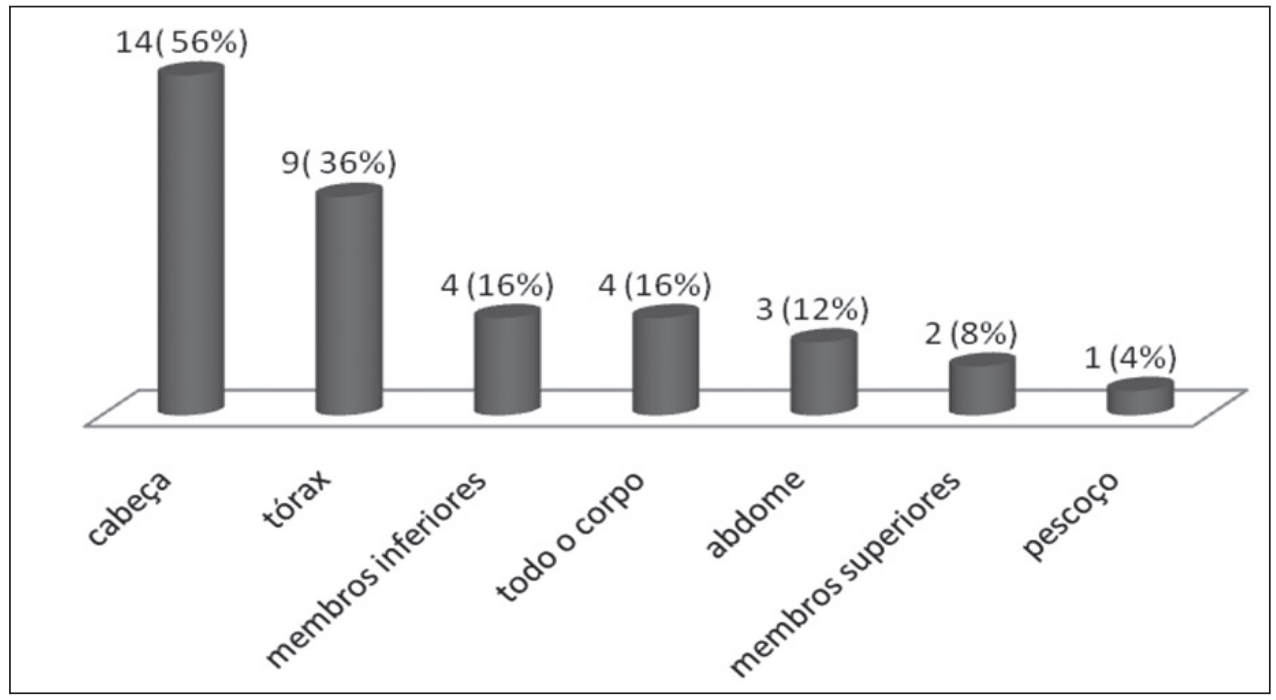

Gráfico 1 - Caracterização das vítimas de AT fatais, segundo parte do corpo atingida Curitiba. SINAN-UST/HT, 2006-2010.

Nos casos de óbitos é assegurado à pensão ao cônjuge ou filhos menores de 18 anos. Já os direitos trabalhistas deverão ser analisados caso a caso, muitas vezes, podendo os familiares receber indenização por danos materiais referentes ao tempo de vida laboral de seu ente ${ }^{(7)}$.

Em estudo realizado em um pronto-socorro do Hospital de Clínicas de São Paulo, em 2007, com pacientes vítimas de violência no trânsito apontou que $60 \%$ dos condutores de veículos a motor estavam em horário de trabalho e $65 \%$ dos condutores de motocicletas estavam no horário de trabalho ou no percurso para o trabalho, o que é caracterizado como $\mathrm{AT}^{(13)}$. Outro estudo realizado em Salvador/Bahia, em 2008, evidenciou também que os acidentes do trabalho de maior gravidade ocorreram no trajeto, envolvendo colisões e atropelamentos, o que confirmam os dados encontrados neste estudo ${ }^{(11)}$.

Constata-se, ainda, como o espaço da rua e o contato direto com o público, permanecem como fatores de risco de acidentes de trânsito e violência em geral. Com relação aos óbitos causados pelo trabalho percebe-se que a violência urbana ganha relevância como fator desencadeante. Estudo realizado em 2004 mostrou que os AT típicos eram decorrentes da violência do trânsito e relacionados à ocupação de seguranças, policiais e vigias. A violência urbana ainda pode contribuir para aumentar os índices de acidentes de trabalho fatais quando os trabalhadores são vítimas de assalto, estupro e projéteis, durante o deslocamento da residência à empresa, caracterizando os AT de trajeto ${ }^{(7)}$.

A violência urbana vitimou 33.619 pessoas em 2003 devido aos acidentes de trânsito, sendo que a taxa de mortalidade foi de 26,5 mortes por acidente de transporte por 100 mil habitantes. A maioria dos eventos (30\%) ocorreu devido a atropelamento de pedestres, seguidos dos ocupantes de veículos (19,3\%). Destaca-se, no entanto, que as ocupações mais atingidas foram os motociclistas (12,9\%), além dos motofretistas e mototaxistas ${ }^{(15)}$. Em estudo realizado em um Pronto-Socorro de um hospital universitário do Rio Grande do Sul, em 2008, sobre acidentes e violências, os AT corresponderam a $7,21 \%$ dos $\operatorname{casos}^{(10)}$. Essa fração é relevante, considerando-se que os AT são todos preveníveis.

Esses dados nos revelam que as ações preventivas em saúde do trabalhador deverão ultrapassar o espaço físico das empresas devido à nova realidade do mercado de trabalho. Para prevenir os AT é necessário investir em educação, além da educação no trânsito, por meio de políticas públicas com campanhas em mídias e a revitalização do transporte coletivo, a fim de evitar os graves AT e suas conseqüências. Além disso, deve-se estimular e apoiar pesquisas que avaliem o comportamento no trânsito, riscos de acidentes devido as condições do veículo e o uso dos equipamentos de proteção individual ${ }^{(15-16)}$.

As quedas, por sua vez, vêm mostrar o não uso dos equipamentos de proteção coletiva (EPC) e individual (EPI), além da negligência às regras 
gerais de segurança no trabalho que deveriam evitar os acidentes e os prejuízos causados aos trabalhadores e as empresas ${ }^{(2,17)}$. Para que isso ocorra é necessário investir em treinamentos para o uso correto de EPI e EPC, além de condições seguras de trabalho como piso antiderrapante, iluminação eficiente e corrimões em locais com risco de quedas.

Outro aspecto a ser destacado é o de que os ferimentos em cabeça, membros superiores e inferiores ocorreram em uma maior freqüência, devido ao fato de estarem relacionados aos acidentes de trânsito e transporte ${ }^{(10)}$ e ao setor indústria, abrangendo a construção civil ${ }^{(17)}$.

\section{CONCLUSÃO}

Considerando os resultados apresentados neste estudo, percebemos a necessidade de serem propostas ações na área de saúde do trabalhador, a fim de prevenir os AT. Para que os AT fatais tornem-se prioridade das políticas públicas de saúde é necessário conhecer sua extensão, não apenas pelos dados informados pelo INSS. Assim, faz se necessário a melhoria das informações de AT no SUS. O SINAN pode ser estratégico como uma ferramenta de coleta de informações para o conhecimento das condições de trabalho da população brasileira, podendo trazer benefícios para a identificação das causas dos AT, sendo esta uma importante etapa para a prevenção deste agravo.

Em relação aos acidentes de trabalho envolvendo a violência no trânsito, outro dado importante desta pesquisa, faz se necessário investimento em políticas públicas educacionais para um trânsito mais seguro e também investimentos no transporte coletivo, a fim de evitar o grande número de acidentes. Porém, também há a necessidade de um trabalho educativo e preventivo que deve ser realizado pelos profissionais de saúde ocupacional, entre eles, o enfermeiro do trabalho, nos ambientes de trabalho.

Outras pesquisas poderão ser realizadas para compreender as contribuições alcançadas por este sistema de informação, bem como, as estratégias educacionais que poderão ser propostas para a melhoria das condições e segurança no trabalho.

\section{REFERÊNCIAS}

1 Ministério da Saúde (BR). Protocolo de Acidente de Trabalho Grave/Fatal / Ministério da Saúde, Secretaria de Atenção à Saúde, Departamento de Ações
Programáticas Estratégicas. Brasília: Ministério da Saúde; 2006.

2 Moraes MVG. Doenças ocupacionais - físico, químico, biológico, ergonômico. São Paulo: Érica; 2010.

3 Brasil. Lei n 5316, de 14 de setembro de 1967: integra o seguro de acidentes do trabalho na previdência social, e dá outras providências. Diário Oficial da União, Brasília(DF), 1967 set 18; Seção 1: 9527.

4. Brasil. Portaria ${ }^{\circ} 777$, de 28 de abril de 2004: dispõe sobre os procedimentos técnicos para a notificação compulsória de agravo à saúde do trabalhador em rede de serviços sentinela específica, no Sistema Único de Saúde-SUS [Internet]. 2004 [citado 2010 set 07]. Disponível em: http://dtr2001.saude.gov.br/ sas/PORTARIAS/port2004/GM/GM-777.htm.

5 Brasil. Portaria 2472, de 31 de agosto de 2010: define as terminologias adotadas em legislação nacional, conforme disposto no Regulamento Sanitário Internacional (RSI 2005), a relação de doenças, agravos e eventos em saúde pública de notificação compulsória em todo território nacional e estabelecer fluxo, critério, responsabilidades e atribuições aos profissionais e serviços de saúde. Diário Oficial União, Brasília(DF), 2010 set 01; Seção 1: 50.

6 Scherer V, Miranda FMD, Sarquis LMM, Lacerda MR. SINAN.NET: um sistema de informação à vigilância na saúde do trabalhador. Cogitare Enferm. $2007 \mathrm{jul} / \mathrm{set}$; $12(3): 330-7$.

7 Hennington EA, Cordeiro R, Moreira DCF. Trabalho, violência e morte em Campinas, São Paulo, Brasil. Cad. Saúde Públ. 2004 mar/abr; 20 (2):610-7.

8 Ministério do Trabalho e Emprego (BR). Superintendência Regional do Trabalho e Emprego do Rio Grande do Sul. Análises de acidentes do trabalho fatais no Rio Grande do Sul: a experiência da Seção de Segurança e Saúde do Trabalhador - SEGUR. Porto Alegre: Superintendência Regional do Trabalho e Emprego do Rio Grande do Sul. Seção de Segurança e Saúde do Trabalhador/SEGUR; 2008.

9 Santana V, Nobre L, Waldvogel BC. Acidentes de trabalho no Brasil entre 1994 e 2004: uma revisão. Ci Saúde Col. 2005 out/nov;10(4):841-55.

10 Hennington EA, Monteiro M. O perfil epidemiológico dos acidentes de trabalho no Vale dos Sinos e o sistema de vigilância em saúde do trabalhador. Hist, Ci, Saúde. 2006 out/dez; 13(4):865-76. 
11 Santana VS, Xavier C, Moura MCP, Espiríto-Santo JS, Araújo,G. Gravidade dos acidentes de trabalho atendidos em serviços de emergência. Rev Saúde Públ. 2009; 45(3):750-60.

12 Santos JLG, Garlet ER, Figueira RB, Lima SBS, Prochnow AG. Acidentes e violências: caracterização dos atendimentos no pronto-socorro de um hospital universitário. Saúde Soc. 2008 jul/ set;17(3):211-8.

13 Anjos KC, Evangelista MRB, Silva JS, Zumiotti AV. Paciente vítima de violência no trânsito: análise do perfil socioeconômico, características do acidente e intervenção do serviço social na emergência. Acta Ortop Bras. 2007;15(5): 262-6.

14 Brasil. Portaria $n^{\circ} 3214$ de 08 de junho de 1978: aprova as Normas Regulamentadoras - NR - do
Capítulo V, Título II, da Consolidação das Leis do Trabalho, relativas a Segurança e Medicina do Trabalho[Internet]. 1978 [citado 2010 set 07]. Disponível em: http://wwwo10.dataprev.gov.br/ sislex/paginas/05/mtb/4.htm

15 Souza ER, Lima MLC. Panorama da violência urbana no Brasil e suas capitais. Ci Saúde Col. 2006;11(Sup):1211-22.

16 Santos AMR, Moura MEB, Nunes BMVT, Leal CFS, Teles JBM. Perfil das vítimas de trauma por acidente de moto atendidas em um serviço público de emergência. Cad. Saúde Públ. 2008 ago;24(8):1927-38.

17 Kirchhof ALC, Magnago TS, Urbanetto JS, Cera MC, Marques CS, Capellari, C. Os acidentes de trabalho atendidos em Pronto Atendimento de Hospital Universitário. Esc Anna Nery. 2003 dez;7(3):361-8.

Endereço do autor / Dirección del autor / Author's address:

Fernanda Moura D'Almeida Miranda

Rua Alfredo José Pinto, 1680, sob 5, Fazendinha

81320-180, Curitiba, PR

E-mail: fernandadalmeida79@hotmail.com 